\title{
The Analysis of Unified Software Engineering Reuse (USER) Using Stable Analytical Model, Design of Structure and Architectural Patterns
}

\author{
Asad Ali, Talha Arshad, Muhammad Hammad, Usman Raza and Farhan Saeed \\ Department of Electrical Engineering, Superior University Lahore, Pakistan
}

\begin{abstract}
The wearisome enthusiasm for making reasonable structures for programming reuse has gone before for whatever period of time that item has existed. Accepting particular intense structures are made for ensuring an abnormal state of reusability from one suspect to the accompanying. The unavoidable slant for dares to ask for extensive alterations, paying little mind to being proposed for most noteworthy reusability, remains strong affirmation of this reality. Programming reusability makes examination of stable examination; arrange, likewise, plan outlines a range of tremendous interest. By extrapolating the unfaltering thoughts that use programming consistent quality show and the Knowledge Maps, we attempt to comprehend programming plans that do not require unrestrained modifications, changes or, then again augment. Such cases works kind of a structure, to the most recent inquiries could be incorporated rely on simply the development of the circumstance to which that is associated. Suitability of such methodology must be displayed in the paper.
\end{abstract}

Key words: USER (the unified software engineering reuse), the software reuse, SSM (software stability model), KMs (knowledge maps), architecture pattern.

\section{Introduction}

Issue with a considerable measure of existing programming outlining illustrations is generally space ward, and using them for totally one of a kind applications could be hard, because a couple of adjustments or changes ought to be made. Using programming strength thoughts to make outlines progresses more noticeable as consistent and quick model, which uses propelling the Business Subjects [5-7], and the Business Objects are [5-7] made recalling the target of a structure. The goal structure, that will shape the foundation of the case occasionally changing, and from now on model delivered that is all the more consistent security, attempt to infer that the illustration it will never going to change, despite the way that is extendable for the utilization in the most vary application autonomous space. The Stable

Corresponding author: Asad Ali, BS electrical engineer, BS student, research field: power.
Pattern [13, 14] constantly outlines thoughts and the speculations.

In the SSM (software stability model), no example is segregated from different outline plus investigation designs.

\section{Stable Analytical Model}

The Stable Analysis Pattern is united in light of the likelihood of an EBT (enduring business theme). These EBTs are inconspicuous, appreciated to a given condition, and fundamentally steady after some time. An EBT is portrayed in the condition of an SAP by utilizing some run of the mill non-unmistakable musings, known as Business Objects. An example of an EBT is the likelihood of notoriety. This idea can be tended as an outline, then in programming, to apply to different settings where notoriety is a required part.

Test Applications in an online business circumstance, for instance, eBay or Amazon, reputation is a fundamental part to complete helpful 
business operation.

Satisfy the requirements. One can in like manner viably watch that the inside classes are shared across over both settings. The Factor helps the Party get notoriety by extended rate.

This SSM (stable software model) can be stood out from conventional exhibit in this circumstance as shown up in Fig. 4. In this show, only the mechanical things are used, which require their substitution in various settings and circumstances.

\section{Stable Design Model}

The standard refinement between a Stable Design Pattern and a Stable Analysis Pattern is that the Design Pattern is locked in or worked from a Business Object, rather than an Enduring Business Theme. Business Objects differ from EBTs in their transitory nature, having a begin and an end. Past this, similar precepts for depiction apply. To speak to Stable
Design Patterns, let us use as an outline influence.

Test Applications consider the occasion of an association picking elevating on the web to affect the methods for overseeing cash of the on the web clients. The business will begin by first orchestrating an agreement with an advancing firm, who will help this endeavor, at a masterminded cost. The circumstance is given in Fig. 6. These circumstances can be appeared differently in relation to a more standard model of a case of effect. Such a traditional model is commonly made quite recently out of the Industrial Objects, most, if not all, apply just to a single circumstance.

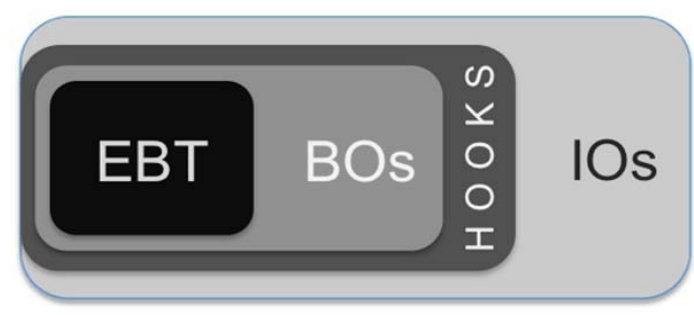

Fig. 1 The layers in EBT and BO's and SSM.

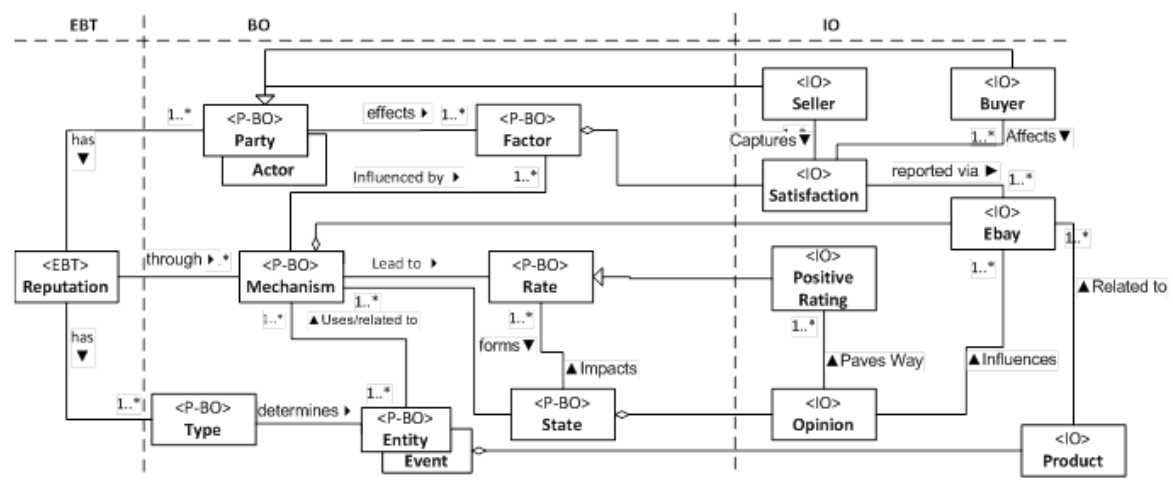

Fig. 2 The reputation analytical pattern for Ebay.

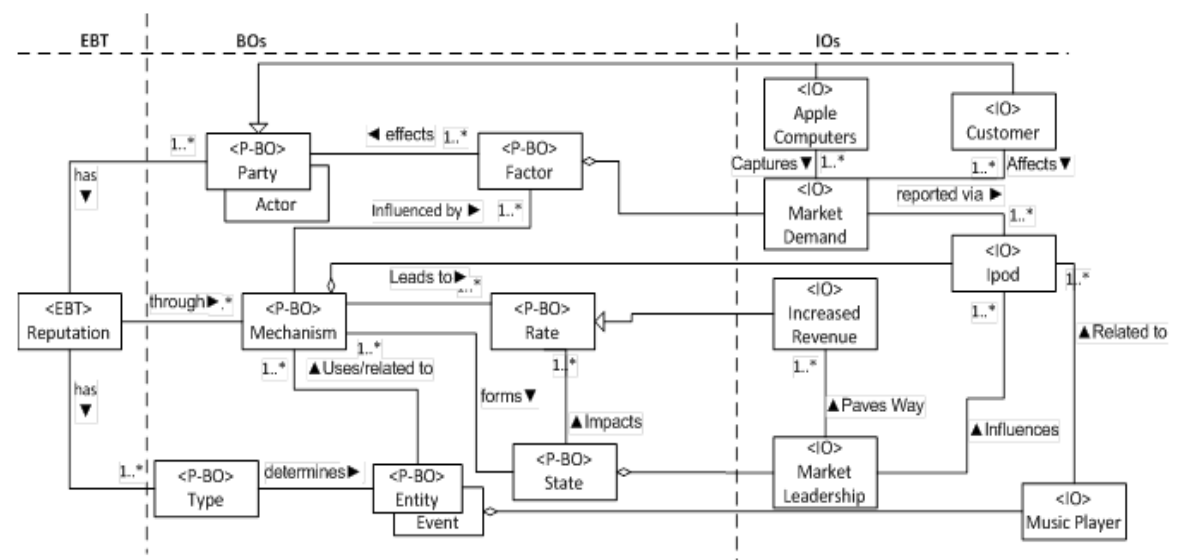

Fig. 3 The reputation analytical pattern for apple iPods. 


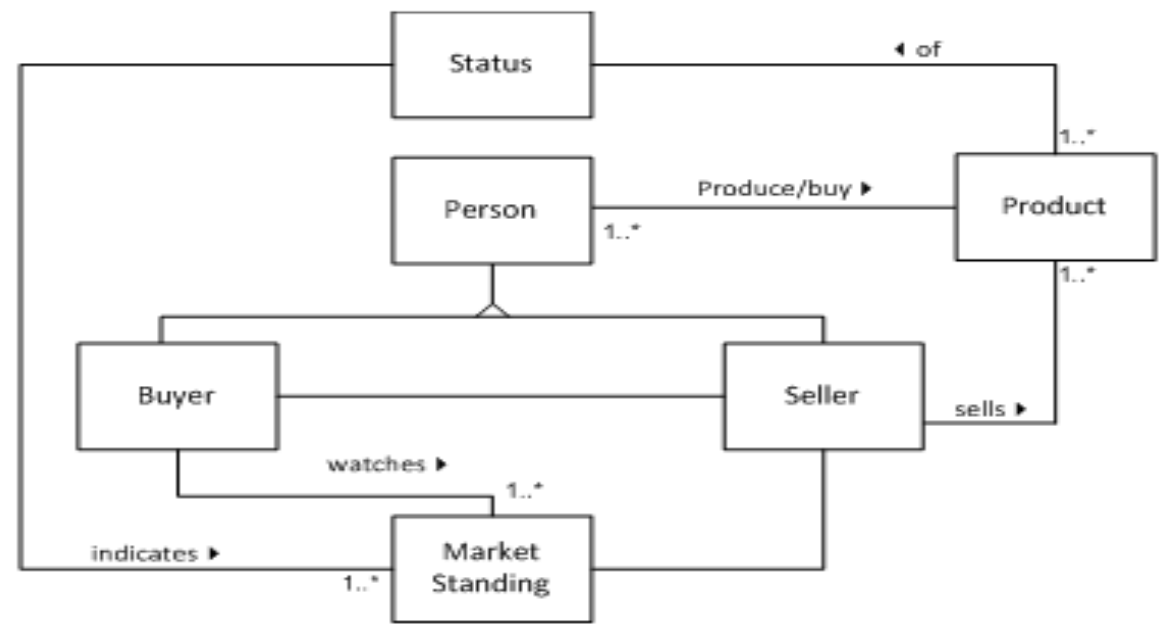

Fig. 4 The traditional model for reputation.

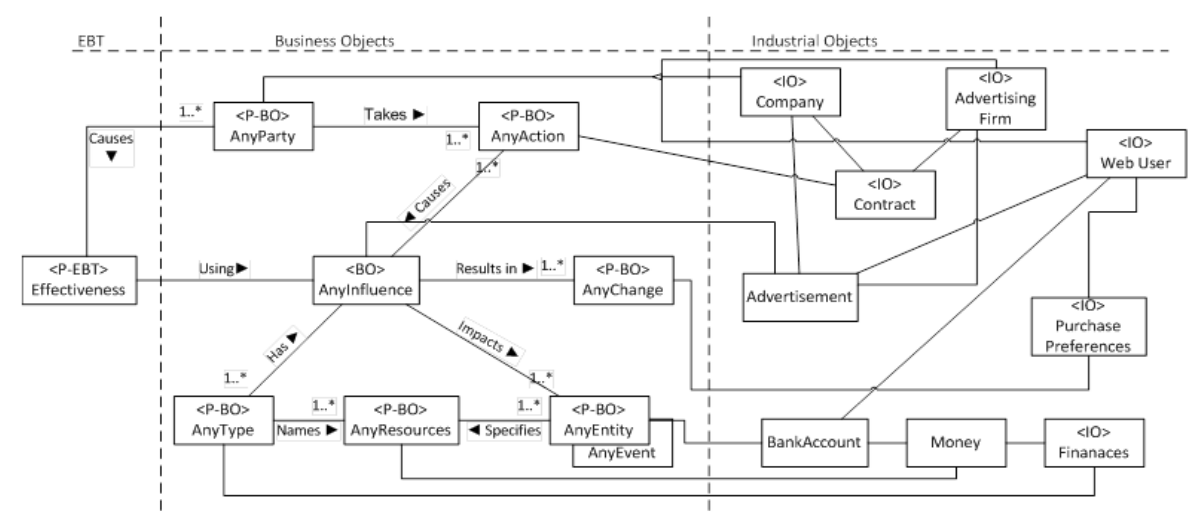

Fig. 5 Influence design pattern for web advertising.

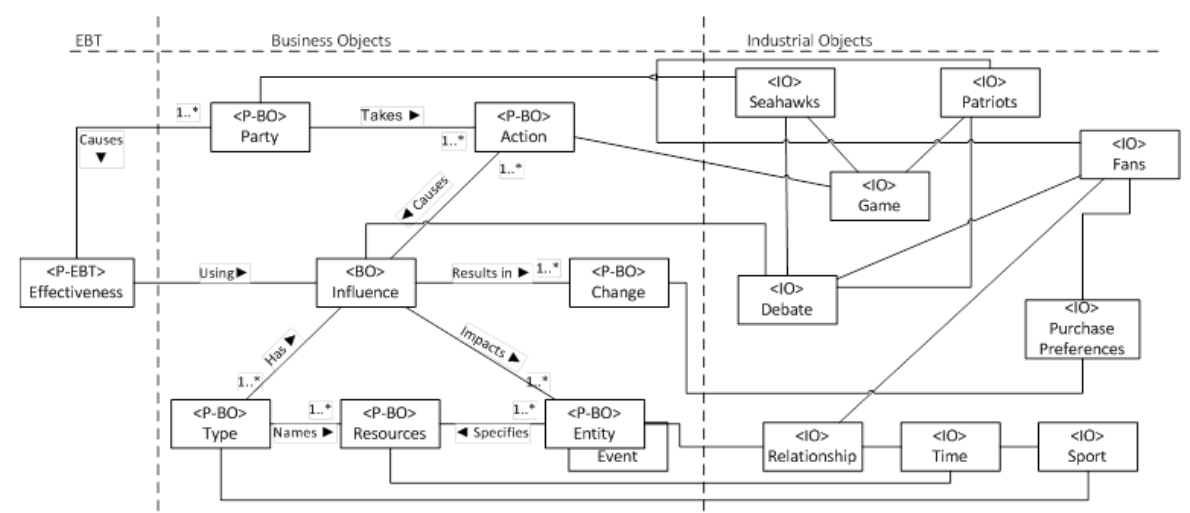

Fig. 6 The influence design in super bowl.

\section{Stable Architecture Model}

Stable Architecture Patterns, can be described as blending of no less than two diagrams or examination plans. This is done similarly as:

By virtue of the vehicle parts deliver strike, the fundamental social affairs included fuse experts and different specialists, the association board people and the laborer's organization. The workers, in light of the effect of development (Factor), exhibit their solicitations to organization and strike and boycott working, unless they are given a raise. The specialist's society, according to the Work Beneficiary Act, goes into course of action for another understanding. 
Table 1 The cyclomatic complexity.

\begin{tabular}{lllll}
\hline Model & $\mathrm{e}$ & $\mathrm{n}$ & $\mathrm{p}$ & $\mathrm{m}$ \\
\hline Traditional influence model & 11 & 12 & 17 & 14 \\
SSM influence design pattern & 6 & 7 & 9 & 4 \\
\hline
\end{tabular}

Table 2 The reusability factor mode.

\begin{tabular}{llll}
\hline Model & cr & ct & rf \\
\hline Traditional influence model & 7 & 12 & $22 \%$ \\
SSM influence design pattern & 8 & 4 & $96 \%$ \\
\hline
\end{tabular}

\section{Analysis of Results}

In the direct representations given above, it should be noticed that the SSMs, however requiring additional initial work than the regular models, are furthermore more comprehensively significant to new and particular settings. For more refined demonstration, an examination of couple of essential estimations might be valuable. There are additional potential estimations, cycloramic disperse quality, for example. This metric is used to choose the interconnectivity of the articles in a given layout or model. The estimations are fused into Table 1.

A more unmistakable cyclic multifaceted nature has different outcomes. In the first place, it shows the multifaceted way of the program itself, as a rule meaning higher upkeep costs. It furthermore shows strong interconnectivity, which makes the program less specific and harder to change and modify. Finally, it furthermore exhibits how much testing is required to be sensibly ensured of the program's authentic execution. For each of these, a humbler cycloramic disperse quality is needed. It is for the most part unmistakably passed on here utilizing SSM.

\section{Conclusion}

With the illustration circumstances and related estimations given above, SSMs are a great deal more adaptable programming game plans when stood out from standard showing frameworks. In spite of the way that we can deliver for the most part versatile models, for instance, the one given in Fig. 3 that can apply to an extent of solidly related circumstances, an all the more intense whole deal game plan, SSM, still remains more noteworthy for a collection of circumstances. In the interim, we see the prerequisite for a couple starting startup expenses of executing the middle classes incorporated at the same time. In any case, the focal point of the program may easily be reused with immaterial or no movements which at last makes this option additionally engaging.

\section{Acknowledgment}

Thanks to Allah for giving me the power to work hard and achieve my goal in life in the form of this paper.

\section{References}

[1] Flood, C. A. "Unified Software Engineering Reuse (USER).” Master thesis, San Jose State University. (In Progress)

[2] Tracz, W. J. 1988. "Software Reuse Myths." ACM Software Engineering Notes 13 (1): 17-21.

[3] Griss, M. L. "Software Reuse: From Library to Factory." Hewlett-Packard Laboratories, 1501 Page Mill Road, Palo Alto, California 94306, USA.

[4] Fayad, M. E., and Altman, A. 2011. "Introduction to Software Stability." Communications of the ACM 44 (9).

[5] Fayad, M. E. 2002. "Accomplishing Software Stability.” Communications of the ACM 45 (1): 95-8.

[6] Fayad, M. E. 2002. "How to Deal with Software Stability." Communications of the ACM 45 (4): 109-12.

[7] Hamza, H. 2002. "A Foundation for Building Stable Analysis Patterns.” Master thesis, University of Nebraska-Lincoln.

[8] Hamza, H. 2002. "Building Stable Analysis Patterns Using Software Stability.” 4th European GCSE Young Researchers Workshop 2002 (GCSE/NoDE YRW 2002), Erfurt, Germany.

[9] Hamza, H., and Fayad, M. E. 2002. "A Pattern Language for Building Stable Analysis Patterns.” 9th Conference on Pattern Language of Programs (PLoP 02), Illinois, USA.

[10] Hamza, H., and Fayad, M. E. 2002. "Model-based Software Reuse Using Stable Analysis Patterns.” ECOOP 2002, Workshop on Model-based Software Reuse, Malaga, Spain.

[11] Mahdy, A., and Fayad, M. E. 2002. "A Software Stability Model Pattern.” Pattern Language of Programs, Monticello (PLoP2002), 2002.

[12] Fayad, M. E., Sanchez, H. A., Hegde, S. G. K., Basia, A., and Vakil, A. 2014. Software Patterns, Knowledge Maps, and Domain Analysis. Boca Raton, FL: Auerbach 


\section{Design of Structure and Architectural Patterns}

Publications.

[13] Fayad, M. E. 2015. Stable Analysis Patterns for Software and Systems. Boca Raton, FL: Auerbach Publications.

[14] Fayad, M. E. 2015. Stable Design Patterns for Software and Systems. Boca Raton, FL: Auerbach Publications.

[15] Fayad, M. E. 2015. Stable Software Architecture on-Demand for Software and Systems. Boca Raton, FL: Auerbach Publications. 